\title{
Papel das intervenções educativas relacionado aos riscos ocupacionais para os
}

\section{profissionais de enfermagem}

\author{
Role of educational interventions related to occupational risks for nursing professionals \\ Papel de las intervenciones educativas relacionadas con los riesgos laborales para los profesionales \\ de enfermeira
}

Recebido: 22/04/2021 | Revisado: 01/05/2021 | Aceito: 02/05/2021 | Publicado: 15/05/2021

\author{
Jucirema Rodrigues Farias \\ ORCID: https://orcid.org/0000-0002-2350-1022 \\ Fundação Santa Casa de Misericórdia do Pará, Brasil \\ E-mail: Jucyfarias32@gmail.com \\ Aurimery Gomes Chermont \\ ORCID: https://orcid.org/0000-0001-8715-3576 \\ Fundação Santa Casa de Misericórdia do Pará, Brasil \\ E-mail: agchermont@gmail.com \\ Silvestre Savino Neto \\ ORCID: https://orcid.org/0000-0002-2350-1022 \\ Fundação Santa Casa de Misericórdia do Pará, Brasil \\ E-mail: Savino@ufpa.br \\ Paulo Cesar Beckman da Silva Junior \\ ORCID: https://orcid.org/0000-0002-4616-2019 \\ Universidade Federal do Amapá, Brasil \\ E-mail: paulocesarbeckman@hotmail.com \\ Jucenira Rodrigues Farias \\ ORCID: https://orcid.org/0000-0003-1006-1053 \\ Secretaria Municipal de Saúde de Belém, Brasil \\ E-mail: f.jucy@yahoo.com.br \\ Raissa dos Santos Flexa \\ ORCID: https://orcid.org/0000-0002-3417-2623 \\ Universidade Federal do Amapá, Brasil \\ E-mail: raissaflexa@gmail.com
}

\begin{abstract}
Resumo
Objetivo: Descrever quais os riscos ocupacionais encontrados no ambiente laboral da enfermagem de acordo com as literaturas, bem como identificar o papel das intervenções educacionais em saúde relacionado aos riscos ocupacionais. Método: revisão integrativa, cujo os critérios de elegibilidade dos artigos foram corresponder aos descritores associados: Profissionais de Enfermagem, Tecnologia Biomédica, Riscos Ocupacionais, Enfermagem do Trabalho e estar disponível eletronicamente nas bases de dados: BVS, LILACS, Medline/PUBMED, SCIELO e na BDENF, com ano de publicação referente a 2015 a 2020. Resultados: foram selecionadas 16 publicações para análise, com predomínio de autores brasileiros enfermeiros, dos quais observou-se a preocupação do Profissional da área quanto a redução e controle dos riscos ocupacionais para com a sua equipe, nos diferentes ambientes de trabalho, sendo encontrado estudos em sua maioria em hospitais seguido por unidades básicas de saúde, utilizando a educação continuada e permanente como ferramenta para minimizar os riscos ocupacionais. Considerações Finais: o profissional de enfermagem compondo a equipe de saúde é o profissional mais exposto aos riscos ocupacionais nos diversos ambientes de prestação de serviços de saúde. Riscos como biológicos, químicos, físicos, ergonômicos e psicossociais.
\end{abstract}

Palavras-chave: Profissionais de enfermagem; Tecnologia biomédica; Riscos ocupacionais; Enfermagem do trabalho.

\begin{abstract}
Objective: To describe the occupational risks found in the nursing work environment according to the literature, as well as to identify the role of educational health interventions related to occupational risks. Method: integrative review, who see ligibility criteria of the articles were correspond to the associated descriptors: Nursing Professionals, Biomedical Technology, Occupational Risks, Occupational Nursing and being available electronically in the following databases: VHL, LILACS, Medline/PUBMED, SCIELO and BDENF, with year of publication for 2015 to 2020. Results: 16 publications were selected for analysis, there was a predominance of Brazilian nursing authors, of which the nurse professional's concern was observed regarding ther eduction and control of occupational risks to his team in different work environments, and studies were found mostly in hospitals followed by basic health units, using continuing and permanent education as a tool to minimize occupational risks. Final Considerations: the nursing professional composing
\end{abstract}


the health team is the professional most exposed to occupational risks in the various environments of health service provision. Risks such as biological, chemical, physical, ergonomic and psychosocial.

Keywords: Nursing professionals; Biomedical technology; Occupational risks; Occupational nursing.

\section{Resumen}

Objetivo: Describir-los riesgos laborales encontrados em el ambiente laboral de enfermería de acuerdo a la literatura, así como identificar el rol de las intervenciones educativas em salud relacionadas con los riesgos laborales. Método: revisión integradora, cuyos criterios de elegibilidad para los artículos fueron coincidir con los descriptores asociados: Profesionales de Enfermería, Tecnología Biomédica, Riesgos Laborales, Enfermería Laboral y estar disponible electrónicamente en las bases de datos: BVS, LILACS, Medline/PUBMED, SCIELO y en BDENF, con año de publicación referido a 2015 a 2020.Resultados:Se seleccionaron16 publicaciones para análisis, con predominio de autores de enfermería brasileños, de los cuales se observó la preocupación del profesional por la reducción y control de riesgos laborales hacia su equipo, en los diferentes ambientes de trabajo, siendo estudios encontrados mayoritariamente en hospitales. Seguido de las unidades básicas de salud, utilizando la educación continua y permanente como herramientas para minimizar los riesgos laborales. Consideraciones finales: El profesional de enfermería que compone el equipo de salud es el profesional más expuesto a riesgos laborales en los diferentes entornos de prestación de servicios de salud. Riesgos como biológicos, químicos, físicos, ergonómicos y psicosociales.

Palabras clave: Profesionales de enfermería; Tecnología biomédica; Riesgos laborales; Trabajo de enfermería.

\section{Introdução}

Os riscos ocupacionais fazem parte da rotina dos profissionais de enfermagem principalmente no ambiente hospitalar, por meio de situações que os expõem podendo levar a riscos físicos, biológicos, mentais e sociais dos mesmos. Decorrem de fatores específicos do ambiente e condições de trabalho, bem como, o tempo de serviço, formação e categoria profissional (Goulart et al, 2020).

Com a incorporação de tecnologias e inovações na assistência a saúde os gestores hospitalares defrontaram-se com os denominados riscos ocupacionais e os agentes de risco nos ambientes de trabalho, incluindo as novas situações também produtoras de perigos e riscos. Neste contexto, existem os riscos potenciais aos profissionais da saúde, em especial, a equipe de enfermagem que de acordo com a atividade específica que desenvolvem estão submetidos em maior ou menor grau a estes riscos (Baltazhar, Marilda, Souza, Cavagna \& Valente, 2017).

Defini-se risco ocupacional todo trabalho e situação que podem desestruturar o plano físico, mental, social dos trabalhadores. Assim, o conjunto de circunstâncias que podem causar efeitos adversos, como morte, ferimentos, doenças ou danos a saúde é definida como uma situação ou fator de risco (Souza, Antunes Cortez, \& Gomes do Carmo, 2017).

Os estudos comprovam que o exercício da enfermagem está associado uma variada exposição a vários fatores de risco, como jornada de trabalho estafantes e o consequente desrespeito ao ciclo circadiano, horários de alimentações inadequados, riscos posturais entre outros. Neste contexto infere-se a preocupação com as condições de trabalho da enfermagem nos hospitais, atraindo a atenção de pesquisadores devido ao risco que o ambiente e atividade oferecem, destacando os riscos mais comumente para prática da enfermagem como os riscos físicos, ergonômicos, biológicos, psicossociais e de acidentes (Silva, Batista de Carvalho, Leite \& Oliveira, 2020)

As atividades da equipe de enfermagem nas instituições hospitalares caracterizam-se, habitualmente, pela prestação do cuidado nas 24 horas do dia, ininterruptamente, permitindo a continuidade da assistência aos pacientes internados, permanecendo grande parte da jornada de trabalho em contato direto com o paciente (Gonçalves, Rocha, Assad, Tavares \& Marta, 2019).

A discursão acerca da preocupação do trabalhador de saúde em relação a sua segurança ocupacional, precisa ser rotineiramente realizada. A elaboração do planejamento de ações de educação permanente que estimulem a construção de conhecimento e reflexão acerca de sua prática profissional pode auxiliar os trabalhadores nesse processo de promoção e prevenção da saúde ocupacional. Assim, precisam ser incentivadas, especialmente ao considerar-se que é a partir da mobilização dos próprios trabalhadores de enfermagem que se pode haver um aprimoramento da percepção acerca dos riscos a que estão expostos e como eles podem e devem ser minimizados. 
Implementar ações educativas por um processo crítico-reflexivo sobre os riscos ocupacionais onde os profissionais de enfermagem estão expostos, pode gerar uma segurança a mais para o profissional. Visto que hábitos preconcebidos podem ser modificados mediante um trabalho em grupo e compartilhamento do conhecimento. Nesse cenário a medida que o conhecimento é construído, favorece a autonomia do trabalhador de enfermagem pois se sente mais inserido no processo laboral e como coresponsável da manutenção de sua saúde (Loro, Bittencourt \& Zeitoune, 2017)

Alguns estudos propõem as seguintes propostas educacionais como: conhecimento de fatores desencadeantes de estresse; mudanças em programas de educação continuada; sensibilizar os profissionais quanto aos riscos ocupacionais, desenvolvimento de um plano estratégico mais humano nas ações de saúde ocupacional; melhorias no ambiente laboral, assim como medidas educacionais que criticam e problematizam a realidade do trabalho. O profissional enfermeiro tem papel relevante no processo de elaboração de boas práticas de biossegurança, promovendo a comunicação da equipe e identificando os gatilhos de riscos ocupacionais que possam afetar a saúde dos mesmos. (Souza et al. 2017).

Diante da contextualização relatada, surgiu o interesse em fazer a revisão integrativa como parte da dissertação de mestrado, buscando identificar recursos educacionais que facilitem o conhecimento e incorporação de boas práticas de biossegurança sobre os riscos ocupacionais.

Nesse sentido esta pesquisa busca descrever quais os riscos ocupacionais encontrados nos diversos ambientes laborais da Enfermagem de acordo com as literaturas? Qual o papel das intervenções educacionais em saúde relacionado aos riscos ocupacionais no trabalho dos Profissionais de Enfermagem?

\section{Metodologia}

Para alcançar o objetivo proposto neste estudo, optou-se pela revisão integrativa de literatura. Por ser um método que reuni e sintetiza resultados de pesquisas sobre um delimitado tema ou questão, de maneira sistemática e ordenada, contribuindo para o aprofundamento do conhecimento do tema investigado (Cecilio \& Oliveira, 2017).

Foram obedecidas as seguintes etapas para elaboração da presente revisão integrativa: -definição do tema e seleção da questão da pesquisa - definição das bases de dados a serem utilizadas; -estabelecimentos de critérios de inclusão e exclusão dos artigos; - definição das informações a serem extraídas dos artigos selecionados; -análise dos resultados; -discussão e apresentação dos resultados (Cecilio \& Oliveira, 2017).

As principais bases de dados eletrônicas utilizadas vinculadas a saúde foram: a Biblioteca Virtual de Saúde (BVS), fontes como LILACS (América Latina e Literatura Caribenha em Ciências da Saúde, Medline/PUBMED (Pesquisa Sistema de Análise online para literatura médica), SCIELO e na BDENF.A consulta foi efetuada no período de 05 de Setembro de 2020 a 26 de Outubro de 2020.

Para facilitar a busca dos artigos construiu-se a estratégia PICO (P=Paciente,I=Intervenção, C=Comparação e $\mathrm{O}=$ Resultado), nesta pesquisa não houve o estudo de Comparação. Onde P= Enfermagem, I=Tecnologias em Saúde, O=Riscos Ocupacionais e Saúde Ocupacional. Assim na busca dos descritores associados, excluindo termos repetidos nos sinônimos e entry-terms. Aplicado os operadores boleanos AND E OR, sendo montado um resumo cruzando os termos para facilitar a busca com os seguintes descritores: Profissionais de Enfermagem, Tecnologia Biomédica, Riscos Ocupacionais, Enfermagem do Trabalho. Como critério de inclusão foram: artigos indexados nas bases referidas escritos em português, inglês, espanhol publicados nos últimos cinco anos (2016-2020) com acesso livre, como filtro empregou-se os textos completos, trabalho com humanos, revisões. Realizou-se uma breve leitura do título e resumo dos artigos, sendo excluídos publicações que não comtemplassem o tema e objetivos da pesquisa em questão e artigos repetidos. 


\section{Resultados}

A amostra inicial foi composta por um total de 1.826 artigos encontradas nas bases de dados online pesquisadas, como amostra final foram selecionados 16 artigos incluindo duas teses de mestrado, dentre estes dois em espanhol e dois em inglês, todos os artigos selecionados foram lidos na íntegra. Para a extração dos dados foi montado uma matriz de síntese englobando as seguintes categorias: Enfermagem, Riscos Ocupacionais, Serviços de Saúde e Intervenções Educativas.

Com base na análise dos estudos realizou-se a caracterização dos artigos a partir dos autores, ano de publicação, objetivo do estudo, metodologia, público alvo e conclusão. Sendo apresentada no Quadro 1 abaixo.

Quadro 1 - Apresentação dos artigos incluídos na revisão integrativa, segundo os autores, ano de publicação, objetivo do estudo, metodologia, público alvo e conclusão.

\begin{tabular}{|c|c|c|c|c|c|}
\hline $\mathbf{N}^{\mathbf{o}}$ & Autor/ano & Objetivo do Estudo & Metodologia & Público Alvo & Principais Conclusões \\
\hline 01 & $\begin{array}{l}\text { Paiva Leite, J., \& Araujo, } \\
\text { G. (2016). }\end{array}$ & $\begin{array}{l}\text { Avaliar a percepção dos } \\
\text { enfermeiros quanto aos riscos } \\
\text { ocupacionais existentes nas } \\
\text { unidades de clínicas médica e } \\
\text { cirúrgica. }\end{array}$ & $\begin{array}{l}\text { Estudo } \\
\text { exploratório- } \\
\text { descritivo, de } \\
\text { natureza } \\
\text { qualitativa }\end{array}$ & Enfermeiros & $\begin{array}{l}\text { Necessidade da educação permanente } \\
\text { a respeito de todos os riscos } \\
\text { ocupacionais existentes no ambiente } \\
\text { de trabalho. }\end{array}$ \\
\hline 02 & $\begin{array}{l}\text { Silva, R., Valente, G., } \\
\text { Barreto, B., \& Camacho, } \\
\text { A. (2016) }\end{array}$ & $\begin{array}{l}\text { Descrever a atuação do } \\
\text { enfermeiro no gerenciamento } \\
\text { dos riscos ocupacionais em } \\
\text { plataformas de petróleo. }\end{array}$ & $\begin{array}{l}\text { Revisão } \\
\text { Bibliográfica } \\
\text { Integrativa,de } \\
\text { abordagem } \\
\text { qualitativa }\end{array}$ & $\begin{array}{l}\text { Trabalhadores de } \\
\text { Plataforma; } \\
\text { Enfermeiro. }\end{array}$ & $\begin{array}{l}\text { Gerenciamento de riscos ocupacionais } \\
\text { é a melhor forma para diminuir e evitar } \\
\text { acidentes de trabalho, ou seja, } \\
\text { acidentes ocupacionais. }\end{array}$ \\
\hline 03 & $\begin{array}{l}\text { Baltazhar, M. A. P.; } \\
\text { Marilda, A. Souza, D.F.; } \\
\text { Cavagna, V. M.; \& } \\
\text { Valente, C.G.S. (2017) }\end{array}$ & $\begin{array}{l}\text { Refletir sobre os riscos } \\
\text { ocupacionais aplicados à } \\
\text { gestão de segurança no } \\
\text { ambiente hospitalar. }\end{array}$ & $\begin{array}{lr}\text { Estudo } & \text { Descritivo } \\
\text { com } & \text { análise } \\
\text { reflexiva } & \end{array}$ & $\begin{array}{l}\text { Profissionais de } \\
\text { Saúde em especial } \\
\text { de Enfermagem. }\end{array}$ & $\begin{array}{l}\text { O reconhecimento dos riscos e a } \\
\text { aplicação das medidas preventivas, } \\
\text { com foco na segurança e saúde do } \\
\text { trabalhador, tragam melhoria na vida } \\
\text { dos profissionais. }\end{array}$ \\
\hline 04 & Melo, C. M. S. S. (2017) & $\begin{array}{l}\text { Elaborar cartilha educativa } \\
\text { acerca de informações } \\
\text { relevantes quanto à exposição } \\
\text { a material biológico. }\end{array}$ & $\begin{array}{l}\text { Estudo de caso } \\
\text { com abordagem } \\
\text { qualitativa. }\end{array}$ & $\begin{array}{l}\text { Equipe } \\
\text { Enfermagem. }\end{array}$ & $\begin{array}{l}\text { Difundir e capacitar os profissionais } \\
\text { para o entendimento dos riscos } \\
\text { ocupacionais potencializando a } \\
\text { implantação de medidas preventivas } \\
\text { junto às instituições }\end{array}$ \\
\hline 05 & $\begin{array}{l}\text { Januário, G., de Carvalho, } \\
\text { P., Lemos, G., Gir, E., \& } \\
\text { Toffano, S. (2017). }\end{array}$ & $\begin{array}{l}\text { Descrever os acidentes } \\
\text { ocupacionais envolvendo } \\
\text { material biológico com } \\
\text { trabalhadores da equipe de } \\
\text { enfermagem. }\end{array}$ & $\begin{array}{l}\text { Estudo descritivo, } \\
\text { retrospectivo, com } \\
\text { abordagem } \\
\text { quantitativa. }\end{array}$ & $\begin{array}{l}\text { Equipe } \quad \text { de } \\
\text { Enfermagem }\end{array}$ & $\begin{array}{l}\text { Evidencia a necessidade de educação } \\
\text { continuada para esses profissionais e a } \\
\text { implantação de dispositivos com } \\
\text { engenharia de segurança. }\end{array}$ \\
\hline 06 & $\begin{array}{l}\text { Souza, V., Antunes } \\
\text { Cortez, E., \& Gomes do } \\
\text { Carmo, T. (2017). }\end{array}$ & $\begin{array}{l}\text { Identificar os riscos } \\
\text { ocupacionais associados aos } \\
\text { trabalhadores de UTI. }\end{array}$ & $\begin{array}{l}\text { Revisão } \\
\text { Integrativa }\end{array}$ & $\begin{array}{l}\text { Equipe } \\
\text { Enfermagem }\end{array}$ & $\begin{array}{l}\text { Todos os riscos ocupacionais estão } \\
\text { presentes na UTI, prevalecendo o risco } \\
\text { ergonômico. }\end{array}$ \\
\hline 07 & $\begin{array}{l}\text { Arcanjo, R., Chistovam, } \\
\text { B., Braga, A., \& Silvino, } \\
\text { Z. (2018). }\end{array}$ & $\begin{array}{l}\text { Mapear o perfil bibliométrico } \\
\text { das produções sobre riscos } \\
\text { ocupacionais na equipe de } \\
\text { enfermagem e identificar as } \\
\text { recomendações apontadas } \\
\text { pelos artigos para redução ou } \\
\text { controle da exposição aos } \\
\text { riscos ocupacionais pela } \\
\text { equipe de enfermagem. }\end{array}$ & $\begin{array}{l}\text { Revisão } \\
\text { integrativa } \\
\text { natureza } \\
\text { qualitativa }\end{array}$ & $\begin{array}{l}\text { Equipe } \quad \text { de } \\
\text { Enfermagem. }\end{array}$ & $\begin{array}{l}\text { O estudo identificou alta frequência } \\
\text { de publicações voltadas para riscos } \\
\text { biológicos, e semelhantemente, o } \\
\text { número de publicações desenvolvidas } \\
\text { em unidades hospitalares foi } \\
\text { expressivo também.Identificou a } \\
\text { intervenção educativa como principal } \\
\text { estratégia para redução dos riscos } \\
\text { ocupacionais. }\end{array}$ \\
\hline 08 & $\begin{array}{l}\text { Loro, M. M.; Bittencourt, } \\
\text { V.L.L.; \& Zeitoune, R. C. } \\
\text { G.;(2017) }\end{array}$ & $\begin{array}{l}\text { Analisar uma prática } \\
\text { educativa acerca dos riscos } \\
\text { ocupacionais, desenvolvida } \\
\text { com uma equipe de } \\
\text { enfermagem que atua em } \\
\text { unidade de emergência } \\
\text { hospitalar. }\end{array}$ & $\begin{array}{l}\text { Pesquisa } \\
\text { qualitativa, } \\
\text { desenvolvida com } \\
\text { a pesquisa } \\
\text { convergente } \\
\text { assistencial. }\end{array}$ & $\begin{array}{ll}\text { Equipe } & \text { de } \\
\text { Enfermagem } & \end{array}$ & $\begin{array}{l}\text { Permitem inferir que os participantes } \\
\text { acumulavam experiências que } \\
\text { influenciavam suas posturas frente } \\
\text { aos riscos do processo de trabalho, o } \\
\text { que os faziam, por vezes, ter uma } \\
\text { conduta de risco. Assim, é necessária } \\
\text { uma ação educativa que estimule a } \\
\text { reflexão, promova autonomia e a } \\
\text { postura protetora de si e do outro. }\end{array}$ \\
\hline
\end{tabular}




\begin{tabular}{|c|c|c|c|c|c|}
\hline 09 & $\begin{array}{l}\text { Arcanjo, R. V. G., } \\
\text { Christovam, B. P., \& } \\
\text { Braga, A. L. de S. (2019). }\end{array}$ & $\begin{array}{l}\text { Levantar os saberes e } \\
\text { práticas dos profissionais de } \\
\text { enfermagem sobre os riscos } \\
\text { ocupacionais en- contrados } \\
\text { nas unidades de atenção } \\
\text { básica ;avaliando o impacto } \\
\text { dessa intervenção educativa. }\end{array}$ & $\begin{array}{l}\text { Estudo quase- } \\
\text { experimental com } \\
\text { desenho pré- } \\
\text { teste/pós-teste } \\
\text { com r grupo } \\
\text { controle não } \\
\text { equivalente com } \\
\text { abordagem } \\
\text { quantitativa }\end{array}$ & $\begin{array}{ll}\text { Equipe } & \text { de } \\
\text { Enfermagem } & \end{array}$ & $\begin{array}{l}\text { A intervenção educativa constitui-se } \\
\text { estratégia eficaz para a aquisição de } \\
\text { saberes e propicia a adoção de práticas } \\
\text { preventivas e de promoção da saúde. }\end{array}$ \\
\hline 10 & Quemel et al (2019) & $\begin{array}{l}\text { Analisar os fatores } \\
\text { relacionados aos Acidentes de } \\
\text { trabalho(AT) entre a categoria } \\
\text { profissionais de enfermagem } \\
\text { em um hospital publico de } \\
\text { grande porte em Belem (PA), } \\
\text { no periodo de janeiro de 2009 } \\
\text { a dezembro de 2016. }\end{array}$ & $\begin{array}{l}\text { Estudo analitico, } \\
\text { retrospectivo e } \\
\text { longitudinal com } \\
\text { abordagem } \\
\text { quantitativa }\end{array}$ & $\begin{array}{ll}\text { Equipe } & \text { de } \\
\text { Enfermagem }\end{array}$ & $\begin{array}{l}\text { As maiores taxas de incidencia de AT } \\
\text { ocorreram nos anos de } 2012(49,5) \text { e } \\
\text { de } 2014(60,2) \text { a cada } 1.000 \\
\text { profissionais por ano. Por mais que } \\
\text { esses resultados se assemelhem a } \\
\text { outros evidenciados e disponiveis na } \\
\text { literatura, particulariza-se o estudo } \\
\text { por se tratar de dados provenientes de } \\
\text { um hospital publico da Região Norte, } \\
\text { área de abrangência que não é } \\
\text { contemplada por estudos dessa } \\
\text { natureza. }\end{array}$ \\
\hline 11 & $\begin{array}{l}\text { Gonçalves, K. O. da S., } \\
\text { Rocha, R. G., Assad, L. } \\
\text { G., Tavares, J. M. A. B., } \\
\text { \& Marta, C. B. (2019). }\end{array}$ & $\begin{array}{l}\text { Avaliar os riscos e as } \\
\text { circunstâncias da ocorrência } \\
\text { de acidentes com material } \\
\text { biológico } \\
\text { trabalhadores de Enfermagem } \\
\text {. }\end{array}$ & $\begin{array}{l}\text { Estudo é } \\
\text { descritivo, } \\
\text { retrospectivo e } \\
\text { quantitativo }\end{array}$ & $\begin{array}{ll}\text { Equipe } & \text { de } \\
\text { Enfermagem. } & \end{array}$ & $\begin{array}{l}\text { Concluiu-se que os profissionais de } \\
\text { enfermagem apresentam dificuldades } \\
\text { de acesso ao serviço de notificação dos } \\
\text { acidentes. Verificou-se a necessidade } \\
\text { de ações de educação permanente } \\
\text { junto aos profissionais de enfermagem } \\
\text { com vistas a minimização do problema } \\
\text { identificado.. }\end{array}$ \\
\hline 12 & $\begin{array}{l}\text { Rodrigues, L. P.; Rezende, } \\
\text { M. P.; Lúcia, A. M. B. S. } \\
\text { Ferreira, A.; \& Goulart, } \\
\text { B.F. (2019) }\end{array}$ & $\begin{array}{l}\text { Investigar o conhecimento e a } \\
\text { adesão do uso dos } \\
\text { equipamentos de proteção } \\
\text { individual pelas equipes de } \\
\text { enfermagem das estratégias } \\
\text { de saúde da família. }\end{array}$ & $\begin{array}{l}\text { Estudo descritivo, } \\
\text { transversal de } \\
\text { abordagem } \\
\text { quantitativa. }\end{array}$ & $\begin{array}{ll}\text { Equipe } & \text { de } \\
\text { Enfermagem } & \end{array}$ & $\begin{array}{l}\text { Conhecimento parcial acerca da } \\
\text { temática e adesão ao uso dos } \\
\text { equipamentos, entretanto, os mais } \\
\text { utilizados foram jaleco e luva. É } \\
\text { necessário realizar educação } \\
\text { permanente para incentivar os } \\
\text { profissionais a utilizarem os } \\
\text { equipamentos na prática profissional. }\end{array}$ \\
\hline 13 & Goulart et al(2020) & $\begin{array}{l}\text { Analisar } \\
\text { a ocorrência de acidentes de } \\
\text { trabalho entre trabalhadores } \\
\text { do Serviço de Atendimento } \\
\text { Móvel de Urgência e a } \\
\text { associação com os riscos } \\
\text { ocupacionais identificados. }\end{array}$ & $\begin{array}{l}\text { Estudo } \\
\text { quantitativo, } \\
\text { exploratório e } \\
\text { descritivo, } \\
\text { transversal. }\end{array}$ & $\begin{array}{l}\text { Condutores de } \\
\text { veículos de } \\
\text { emergência, } \\
\text { técnicos e } \\
\text { auxiliares de } \\
\text { enfermagem, } \\
\text { enfermeiros } \\
\text { médicos. }\end{array}$ & $\begin{array}{l}\text { As ocorrências de acidentes de } \\
\text { trabalho durante as atividades no } \\
\text { serviço pré-hospitalar estão } \\
\text { relacionadas à categoria profissional } \\
\text { dos trabalhadores, mesorregião de } \\
\text { atuação, afastamentos do trabalho e a } \\
\text { exposição dos trabalhadores a } \\
\text { distintos riscos ocupacionais. }\end{array}$ \\
\hline 14 & $\begin{array}{l}\text { Silva, J., Batista de } \\
\text { Carvalho, A., Leite, H., \& } \\
\text { Oliveira, E. (2020). }\end{array}$ & $\begin{array}{l}\text { Refletir sobre os riscos } \\
\text { ocupacionais que envolvem } \\
\text { os trabalhadores da } \\
\text { saúde na nova pandemia de } \\
\text { coronavírus (COVID-19). }\end{array}$ & $\begin{array}{l}\text { É um ensaio } \\
\text { teórico-reflexivo } \\
\text { qualitativo, com } \\
\text { base na literatura } \\
\text { nacional e } \\
\text { internacional }\end{array}$ & $\begin{array}{l}\text { Trabalhadores de } \\
\text { saúde. }\end{array}$ & $\begin{array}{l}\text { É essencial conhecer e controlar os } \\
\text { riscos aos quais os profissionais de } \\
\text { saúde estão expostos e estabelecer } \\
\text { estratégias de prevenção e } \\
\text { minimização de doenças. }\end{array}$ \\
\hline 15 & Fonseca et al (2020) & $\begin{array}{l}\text { Analisar a associação entre os } \\
\text { riscos ocupacionais e os danos } \\
\text { relacionados ao trabalho de } \\
\text { enfermagem em sala de } \\
\text { vacinação }\end{array}$ & $\begin{array}{l}\text { Estudo transversal } \\
\text { analítico. }\end{array}$ & $\begin{array}{ll}\text { Equipe } & \text { de } \\
\text { Enfermagem }\end{array}$ & $\begin{array}{l}\text { As condições de trabalho a que os } \\
\text { profissionais da enfermagem são } \\
\text { expostos nas salas de vacinação, } \\
\text { expressadas em riscos ocupacionais, } \\
\text { são associadas a danos à sua saúde. }\end{array}$ \\
\hline 16 & Lima et al (2019) & $\begin{array}{l}\text { Descrever o ambiente de } \\
\text { trabalho e reconhecer os } \\
\text { riscos ocupacionais a que a } \\
\text { equipe do Consultório na Rua } \\
\text { está exposta, assim como as } \\
\text { medidas preventivas } \\
\text { aplicáveis segundo a } \\
\text { percepção dos profissionais } \\
\text { integrantes da equipe. }\end{array}$ & $\begin{array}{l}\text { Pesquisa } \\
\text { qualitativa, } \\
\text { exploratória, } \\
\text { descritiva. }\end{array}$ & $\begin{array}{l}\text { Representantes } \\
\text { das áreas de } \\
\text { enfermagem , } \\
\text { serviço social, } \\
\text { terapia } \\
\text { ocupacional } \\
\text { agentes sociais }\end{array}$ & $\begin{array}{l}\text { O estudo permitiu conhecer e } \\
\text { problematizar a exposição a riscos } \\
\text { ocupacionais das equipes do } \\
\text { Consultório na Rua estão expostas, } \\
\text { contribuindo para compreender o } \\
\text { processo e ambiente de trabalho como } \\
\text { os riscos ocupacionais. }\end{array}$ \\
\hline
\end{tabular}

Fontes: Autores (2021). 


\section{Discussão}

Os estudos em língua portuguesa que foram em sua maioria, são mais enfáticos quanto a temática riscos ocupacionais. O interesse pela temática acredita-se ser pela falta de condições estruturais físicas das unidades de saúde, déficit de logística de recurso material, dimensionamento de recursos humanos insuficiente principalmente as vinculadas ao setor público, pois normalmente possuem estruturas físicas precárias ás vezes com adaptações inadequadas, mobílias danificadas e desabastecimento de Equipamentos de Proteção Individual (EPI) o que implica em maior exposição aos riscos ocupacionais.

No quadro acima identificou-se nos artigos, predominância de autoria dos Enfermeiros perfazendo $100 \%$ dos artigos selecionado, percebendo-se a preocupação destes profissionais com seu ambiente laboral. Este dado correlaciona-se a questão de o Pessoal de Enfermagem ser o profissional mais exposto nos ambientes de saúde, principalmente hospitalar a uma gama de riscos ocupacionais durante 24horas de sua atuação, segundo Loro et al, (2017). Dentre o grupo de profissionais que compõe a equipe de saúde a enfermagem é a equipe mais exposta aos riscos ocupacionais rotineiramente, relacionado ao contato direto destes durante a assistência aos pacientes, além do tipo e frequência de assistência prestada (Arcanjo, Christovam \& Braga, 2019).

Quanto aos objetivos descritos nos artigos manifesta-se preocupação do Profissional Enfermeiro quanto a redução e controle dos riscos ocupacionais para com a sua equipe, em relação aos diferentes ambientes de trabalho laboral, associação de riscos ocupacionais e a prevalência de agravos e surgimento de doenças ocupacionais, riscos ocupacionais mais frequentes para enfermagem, acidentes ocupacionais mais descritos na literatura para enfermagem, importância do uso de Equipamento de Proteção Individual, conhecimento da enfermagem sobre os riscos ,bem como a reflexão do profissional de enfermagem no processo de prevenção da saúde ocupacional.

Com relação ao delineamento metodológico oito artigos correspondendo a 50\% dos estudos são de abordagem quantitativa e outros 50\% qualitativos, não foi observado nenhum artigo quanti-qualitativo. Os métodos utilizados nas produções científicas foram bem diversificados sendo apresentados no quadro I acima.

Nos estudos as categorias profissionais de prevalência foi a equipe de enfermagem (dez estudos), apenas um estudo teve como população alvo apenas o Profissional Enfermeiro, demais incluíram além da enfermagem outras categorias (cinco estudos).

As principais conclusões identificadas nos artigos inferem sobre a importância do conhecimento sobre os riscos ocupacionais pelos profissionais de saúde, com atenção aos profissionais de enfermagem visto serem a equipe mais sujeita aos riscos. Reforça também sobre a necessidade destes profissionais serem envolvidos em atividades educativas no ambiente laboral, como a educação permanente e educação continuada em saúde, por acreditar que facilita o aprendizado do profissional em adotar uma boa prática quanto as medidas de vigilância e prevenção aos riscos ocupacionais.

Desse modo, pensa-se que implementar ações educativas a partir de um processo crítico-reflexivo sobre os riscos ocupacionais a que os profissionais de enfermagem estão expostos tem o potencial de gerar um comportamento seguro e eficaz. Nesse ínterim é importante estimulá-los a uma ação crítico-reflexiva e participativa, integrando o trabalhador no processo, sendo o sujeito participativo do mesmo. Á medida que os saberes são compartilhados, constrói-se um novo saber e esse processo favorece a autonomia do trabalhador de enfermagem. Assim, a educação constitui-se em uma estratégia para que o indivíduo tenha mais capacitação e possibilidade de construir-se no mundo do trabalho (Loro et al 2017).

Com vistas à prevenção e/ou minimização dos riscos ocupacionais, deve-se criar no contexto laboral, dentre outras medidas, a educação permanente, recursos humanos adequados, equipamentos e mobiliários apropriados, espaços para reflexão coletiva entre os trabalhadores acerca do seu processo de trabalho.Assim, pode-se inferir que as condições de trabalho às quais os profissionais da enfermagem são expostos nas Sala de Vacina, expressas em riscos ocupacionais, afetam negativamente a sua saúde. Faz-se necessário construir estratégias de educação permanente relacionadas aos riscos ocupacionais, visando a redução dos problemas que acometem a equipe de enfermagem (Fonseca et al, 2020). 
Percebeu-se que o conhecimento profissional a respeito dos riscos ocupacionais é resultado da prática diária e não vêm de pesquisa de campo ou treinamento. Desse modo, faz não se tornar uma ação preventiva. Uma das principais contribuições desta pesquisa é a necessidade de desenvolver programas que tornem as ações preventivas em hábitos. Conhecendo os fatores de riscos, cabe aos gestores iniciar um trabalho no sentido de orientar o trabalhador para evitar os riscos ocupacionais, fornecendo ferramentas necessárias ao seu controle. Portanto implementar um processo contínuo educacional com treinamento específico para saúde dos profissionais poderá fornecer uma melhor compreensão sobre saúde ocupacional e também contribuir na redução ou eliminação dos riscos. (Lima et al, 2019).

Medidas de cunho educativo podem ser uma alternativa para melhoria do serviço prestado por esses sujeitos no que se refere á adesão. Portanto, é primordial a introdução de ações educativas na rotina dos profissionais, no que diz respeito ás normas de biossegurança, devendo ser oferecidos aulas, cursos, seminários, workshops com o objetivo de atingir a compreensão de todos, garantindo uma sociedade mais consciente dos seus direitos e deveres para consigo e com os demais (Santos, 2017).

\section{Considerações Finais}

Neste estudo foi identificado um bom arsenal de publicações principalmente de autores brasileiros, no geral escrito por profissionais enfermeiros, sendo os artigos selecionados em sua maior parte foram do ano de 2017, por melhor corresponderem aos descritores da pesquisa. Os resultados da pesquisa permitem inferir que o profissional de enfermagem compondo a equipe de saúde é o profissional mais exposto aos riscos ocupacionais nos diversos ambientes de prestação de serviços de saúde. Riscos estes como biológicos, químicos, físicos, ergonômicos e psicossociais. A maior exposição há um risco ocupacional depende muito do ambiente laboral e ás vezes observa-se diferenças de exposição nos diferentes ambientes que compõem uma estrutura organizacional de saúde. Percebe-se nos artigos que o conhecimento sobre os riscos ocupacionais facilita a adesão as normas de segurança pelos profissionais de enfermagem, daí a importância da educação continuada e permanente como facilitadora do aprendizado. Pontua-se também que além do conhecimento, estes profissionais precisam ser inseridos em reflexões sobre suas atividades laborais para garantir uma prática prevencionista que perpasse o cotidiano de sua práxis, enfatizando

\section{Referências}

Arcanjo, R., Chistovam, B., Braga, A., \& Silvino, Z. (2018). Gerenciamento dos riscos ocupacionais da enfermagem na atenção básica: estudo exploratório descritivo / Management of occupational risks of nursing in primary health care: a descriptive exploratory study. Revista de Pesquisa: Cuidado é Fundamental Online, 10(2), 351-357. http://dx.doi.org/10.9789/2175-5361.2018.v10i2.351-357

Arcanjo, R. V. G., Christovam, B. P., \& Braga, A. L. de S. (2019). Recomendações sobre exposição aos riscos ocupacionais pela equipe de enfermagem: uma revisão integrativa. Revista Enfermagem Atual In Derme, 83(21). https://doi.org/10.31011/reaid-2017-v.83-n.21-art.291

Baltazhar, M. A. P., Marilda, A. Souza, D.F., Cavagna, V. M., \& Valente, C.G.S. (2017) Gestão dos riscos ocupacionais nos serviços hospitalares: uma análise reflexiva. Rev. enferm UFPE, 11, e9, 10.5205/reuol.11088-99027-5-ED.1109201720.

Cecilio, H. P. M., \& Oliveira, D. C., (2017) Modelos de revisão integrativa: discussão na pesquisa em Enfermagem. Investigação Qualitativa em Saúde//InvestigaciónCualitativaenSalud.

Florêncio, F. C., Nunes Neto, G. V., Silva, Y. L. F. \& Guerra, M. C. G. C. (2018). Riscos ocupacionais evidenciados nos profissionais de enfermagem inseridos nas unidades de urgência e emergência. Enfermagem Brasil,17(5)

Fonseca, E., Sousa, K., Nascimento, F., Tracera, G., Santos, K., \& Zeitoune, R. (2020). Riscos ocupacionais na sala de vacinação e suas implicações à saúde do trabalhador de enfermagem [Occupational risks in the vaccination room and its implications for the health of the nursing workers] [Riesgos laborales en la sala de vacunación $\mathrm{y}$ sus implicaciones para la salud del trabajador de enfermería]. Revista Enfermagem UERJ, 28, e45920. doi:https://doi.org/10.12957/reuerj.2020.45920

Gonçalves, K. O. da S., Rocha, R. G., Assad, L. G., Tavares, J. M. A. B., \& Marta, C. B. (2019). Riscos e circunstâncias de acidentes com material biológico com o trabalhador de enfermagem. Revista Enfermagem Atual In Derme, 87(25). https://doi.org/10.31011/reaid-2019-v.87-n.25-art.195

Goulart, L. S., Rocha, L. P., Carvalho, D. P., Tomaschewski-Barlem, J. G., Dalmolin, G. L., \& Pinho, E. C. (2020). Acidentes de trabalho e os riscos ocupacionais identificados no Serviço de Atendimento Móvel de Urgência. Revista da Escola de Enfermagem da USP, 54, e03603. https://doi.org/10.1590/s1980220x2018056903603

Januário, G., de Carvalho, P., Lemos, G., Gir, E., \& Toffano, S. (2017). Acidentes Ocupacionais Com Material Potencialmente Contaminado Envolvendo Trabalhadores De Enfermagem. Cogitare Enfermagem, 22(1). http://dx.doi.org/10.5380/ce.v22i1.48893 
Lima, A. F. S., Almeida, L. W. de S., Costa, L. de M. C., Marques, E. S., Lima Júnior, M. C. F. \& Rocha, K. R. da S. Lima. (2019). Reconhecimento dos riscos no trabalho do Consultório na Rua: um processo participativo. Revista da Escola de Enfermagem da USP, 53, e03495. https://dx.doi.org/10.1590/s1980$220 \times 2018022603495$

Loro, M. M., Bittencourt, V. L. L., \& Zeitoune, R. C. G.,(2017) Pesquisa convergente assistencial: equipe de enfermagem compartilhando saberes sobre riscos ocupacionais e propondo intervenções. Rev Min Enferm.

Melo, C. M. S. S. (2017) Saúde do trabalhador em ambiente com exposição a material biológico: uma produção tecnológica. Dissertação (Mestrado Profissional em Enfermagem Assistencial) - Universidade Federal Fluminense.

Nazario, E. G., Camponogara, S. \& Dias, G. L. (2017). Riscos ocupacionais e adesão a precauções-padrão no trabalho de enfermagem em terapia intensiva: percepções de trabalhadores. Revista Brasileira de Saúde Ocupacional, 42, e7. https://doi.org/10.1590/2317-6369000009216

Paiva Leite, J., \& Araujo, G. (2016). Riscos Ocupacionais: Percepção De Enfermeiros De Um Hospital Público. Revista Enfermagem Contemporânea, 5(2). http://dx.doi.org/10.17267/2317-3378rec.v5i2.1055

Pimenta, G., Perez Junior, E., Pires, A., Gomes, H., Thiengo, P., \& Paula, V. (2018). Influência da precarização no processo de trabalho e na saúde do trabalhador de enfermagem. Revista de Enfermagem da UFSM, 8(4), 758-768. https://doi.org/10.5902/2179769230180

Quemel, G. K. C., Paula, L. C. C., Peixoto, I. V. P. Peixoto, O. S., Araújo, J.S, \& Pamplona, M. C. C.A. (2019) Fatores relacionados a acidentes de trabalho entre profissionais de enfermagem de um hospital público na Região Norte do Brasil entre os anos de 2009 a 2016. RevBrasMed

Rodrigues, L. P., Rezende, M. P., Lúcia, A. M. B. S. Ferreira, A., \& Goulart, B.F. (2019) Conhecimento e adesão da equipe de enfermagem aos equipamentos de proteção individual. REME - Rev Min Enferm. 10.5935/1415-2762.20190073

Santos, R. S. (2017) Acidentes com materiais perfurocortantes: estratégias educativas para redução de riscos ocupacionais. Dissertação (Mestrado profissional em Saúde e Tecnologia no Espaço Hospitalar) - Universidade Federal do Estado do Rio de Janeiro,

Silva, R., Valente, G., Barreto, B., \& Camacho, A. (2016). O gerenciamento de riscos ocupacionais e as interferências na saúde do trabalhador: revisão integrativa The risk management and interference in occupational health worker: integrative review. Revista de Pesquisa: Cuidado é Fundamental Online, 8(2), 4168-4185. doi:http://dx.doi.org/10.9789/2175-5361.2016.v8i2.4168-4185

Silva, R., Madeira, M., Fernandes, M. A., Batista, O., de Brito, B., \& de Carvalho, N. (2017). Occupational risk between nursing workes in Intensive Therapy Unit. Revista brasileira de medicina do trabalho: publicacao oficial da Associacao Nacional de Medicina do Trabalho-ANAMT, 15(3), 267-275. https://doi.org/10.5327/Z1679443520170027

Silva, R. M. da, Zeitoune, R. C. G., Beck, C. L. C., Martino, M. M. F. de \& Prestes, F. C. (2016). The effects of work on the health of nurses who work in clinical surgery departments at university hospitals. Revista Latino-Americana de Enfermagem, 24, e2743..https://doi.org/10.1590/1518-8345.0763.2743

Silva, J., Batista de Carvalho, A., Leite, H., \& Oliveira, E. (2020). Reflexiones sobre los riesgos ocupacionales en trabajadores de salud en tiempos pandémicos por COVID-19. Revista Cubana De EnfermeríA, 36(2).

Souza, V., Antunes Cortez, E., \& Gomes do Carmo, T. (2017). Medidas educativas para minimizar os riscos ocupacionais na equipe de enfermagem da UTI Educational measures to minimize occupational hazards in the ICU nursing staff. Revista de Pesquisa: Cuidado é Fundamental Online, 9(2), 583-591. doi:http://dx.doi.org/10.9789/2175-5361.2017.v9i2.583-591

Vieira, K. M. R., Vieira Jr, F. U. \& Bittencourt, Z. Z. L. C. (2020). Subnotificação de acidentes de trabalho com material biológico de técnicos de enfermagem em hospital universitário. Rev baiana enferm. 34 (37056). 